ABCDDV/1264

ABCD Arq Bras Cir Dig

2016;29(4):293-212

DOI: /10.1590/0102-6720201600040020

\section{SYNCHRONOUS ANAL MELANOMA AND COLON ADENOCARCINOMA: CASE REPORT AND REVIEW OF DIAGNOSIS AND MANAGEMENT}

Melanoma anal e adenocarcinoma de colon sincrônicos: relato de caso e revisão de diagnóstico e conduta

Eduardo Henrique PIROLLA' ${ }^{1}$, Felipe Piccarone Gonçalves RIBEIRO', Fernanda Junqueira Cesar PIROLA ${ }^{1}$, Camila COSMO $^{2}$, Melany Di BIASI ${ }^{3}$

From the ${ }^{1}$ Harvard Medical School, Boston, Massachusetts, USA ${ }^{2}$ Postgraduate Program in Interactive Process of Organs and Systems, Federal University of Bahia, Salvador, Bahia, Brazil; and ${ }^{3}$ Center for Technological Innovation in Rehabilitation, Federal University of Bahia, Salvador, Bahia, Brazil

HEADINGS - Melanoma. Adenocarcinoma. Diagnosis DESCRITORES - Melanoma. Adenocarcinoma. Diagnóstico

\section{Correspondence:} E-mail: eduardopirolla@gmail.com $\begin{array}{r}\text { Received for publication: 04/02/2016 } \\ \text { Accepted for publication: 02/08/2016 }\end{array}$

Financial source: none Conflicts of interest: none
Eduardo Henrique Pirolla

\section{INTRODUCTION}

M alignant anal melanoma is a rare disorder corresponding to $0.05-1.0 \%$ of all anorectal tumors, and $0.4-1.6 \%$ of all other melanomas ${ }^{7,8,9}$ Its rarity can be confirmed by the fact that for every anal melanoma, there are eight squamous cell carcinomas and 250 anal adenocarcinomas ${ }^{8,9}$.

The article's aim is to present a case of a malignant anal melanoma coexisting with colon adenocarcinoma, in addition to a discussion on how to speed up the diagnosis with simple routine measures, and report an objective treatment.

\section{CASE REPORT}

A 57-year-old patient was admitted with weakness, pale skin and a lump in the inguinal region. According to his medical history, one year prior to admittance the patient was treated for anemia. At the time, he presented positive fecal occult blood test. Endoscopy and contrast exams were normal and no weight loss or changes in bowel movements were noticed.

In the six months prior to admittance, the patient felt sporadic pain in the anal canal that ceased with the use of NSAID suppositories. After 30 days, he sought medical attendance and underwent proctosigmoidoscopy and colonoscopy, along with biopsy of lesions in the anal canal and cecum. The patient was then diagnosed with poorly differentiated carcinoma of the canal and well differentiated tubular adenocarcinoma of the cecum. At the time, a lump began forming in the root of the thigh, just below the inguinal fold.

The patient was refered to Sírio-Libanês Hospital in São
Paulo, Brazil, for surgical treatment of the colon lesion and clinical treatment of the anal canal lesion (Figure 1), as he refused to operate the anal lesion. Abdominal ultrasonography and thorax tomography did not reveal any findings. Surgery was then performed with right ileocecal colectomy, intraoperatory biopsy of lesion in the anal canal and of the right inguinal lymph node.

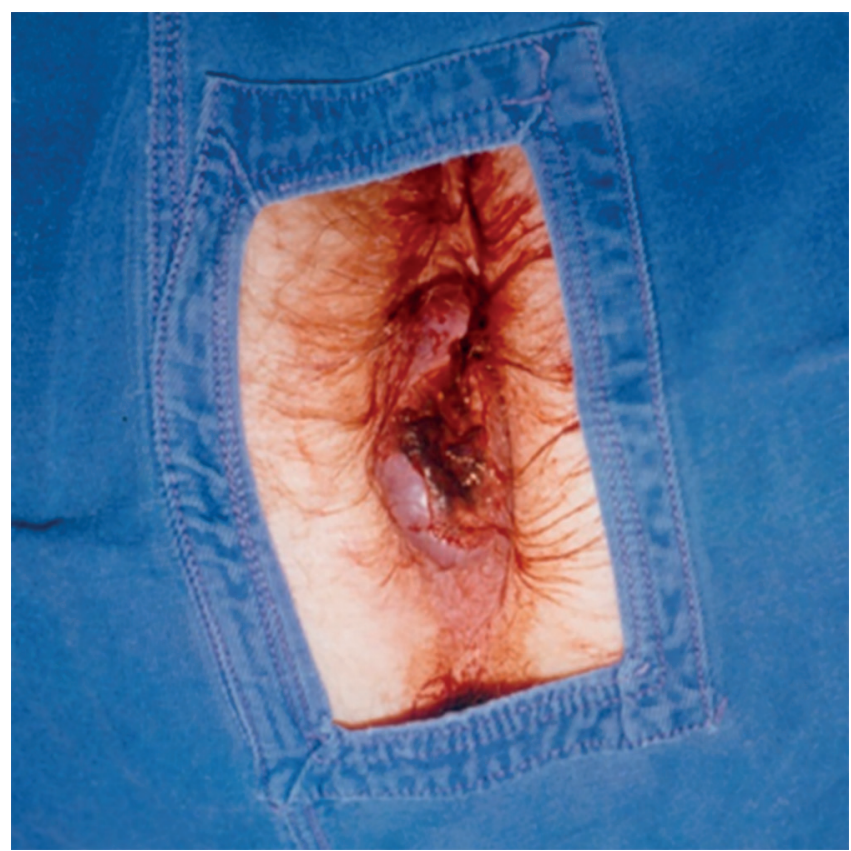

FIGURE 1 - Circunferencial anal lesion in all anorectal canal

Anatomopathological findings of the anal canal revealed an amelanotic malignant melanoma with extensive infiltration and presence of ulcerations and necrosis (Figure 2); right inguinal lesion was a metastatic amelanotic malignant melanoma.

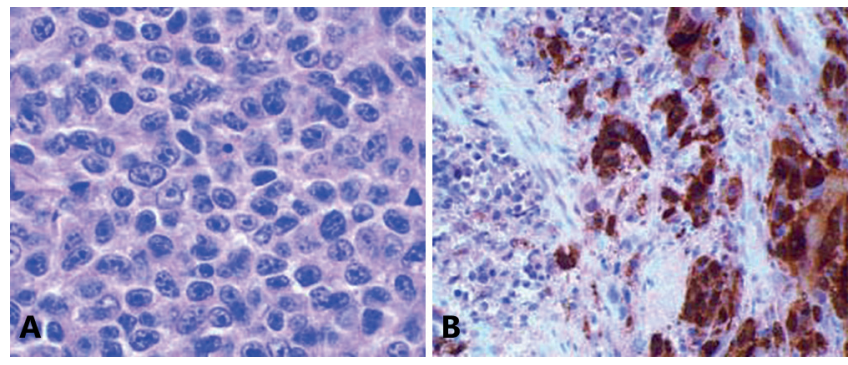

FIGURE 2 - A) Amelanotic epithelioid type (H\&E) and B) epithelioid/lymphoma-like (Study S-100 protein)

Findings on the colon lesion revealed a segment of the left colon had histological stage II invasive tubular adenocarcinoma, a presence of $20 \%$ of mucous membrane, diffuse and extensive infiltration of the colon wall up to the peripheral adipose tissue, ulcerous lesion with irregular areas and necrosis, discreet fibrosis and inflammatory infiltration, and absence of vascular or peri-neural invasion. The anatomopathological examination of surgical borders revealed absence of neoplasm. Assessment of mesocolic lymph nodes exhibited reactive lymphoid hyperplasia with no metastasis $(0 / 13)$.

The patient refused surgical treatment on the anal canal and neglected follow-up. After two months, he returned with exophytic lesion and bleeding, along with significant loss of weight and pain. In the 30 days before hospitalization he presented important anal bleeding with spontaneous remission. In the $6^{\text {th }}$ day prior to hospitalization, there was a new episode of bleeding, which led to hospitalization. A new 
abdominal tomography showed multiple adenomegalies in the paraaortic, paracaval, inguinal, and bilateral perirectal lymph nodes. It also revealed a heterogenic mass with imprecise limits, compatible with ganglion conglomerates of mesenteric origin, next to the hepatic angle of the colon; presence of a soft tissue mass in the anal canal, extending to the ischiorectal fossa towards the perineal border, predominantly on the left, with no dividing plane with the elevator muscle of the anus. Due to the bleeding and ulceration, a colonostomy was performed and local radiotherapy began. After the third session of 300 rads in each there was significant improvement of the bleeding, which stopped after the fourth session. There was clinical improvement and the patient is currently undergoing monitoring.

\section{DISCUSSION}

Malignant anorectal melanoma was first described by Moore in $1857^{1}$. The origin of the tumor appears to be ectodermic, although the presence of melanocytes in the colorectal, above the squamous and transitional regions of the anal canal, proves that the tumor can originate above just as bellow the pectinate line ${ }^{1,2,11}$. Some authors argue that, in almost all cases, the tumor originates in the pectinate line and grows in the submucous space, scrolling through the tissue to emerge on the mucosa at a higher point, thus simulating a primary rectal tumor ${ }^{2,6}$.

The prognosis is poor since the survival rate is $l o w^{5,8,13}$. Some authors relate this to a late diagnosis in most cases ${ }^{1,3,12}$.

The early signs and symptoms are similar to other colorectal diseases, being most frequent anal bleeding, local pain, changes in bowel habits, and in many times, the growth of lumps in the region ${ }^{1,3,8,12}$. The difficulty in diagnosing is brought by the similarities of the symptoms with hemorrhoidal disease or anal fissure, as well as the fact that in $16-41 \%$ of lesions lack pigmentation ${ }^{1,8,12}$.

The incidence is greater in women ${ }^{1,8,12}$. The average age group consists of patients in their sixties and seventies ${ }^{5,6,12}$.

Resection is the treatment of choice among the authors depending on the size of the lesion. The level of infiltration and presence of metastases are some of the factors considered when choosing the extension of resection ${ }^{1,9}$. Previous or adjuvant postoperative radiotherapy and chemotherapy is very debatable.

Many authors believe that the origin of these tumors is in the dentate line, where melanocytes have been detected ${ }^{1,2,8}$. Iron ions have been used for staining, currently; the MassonFontana technique is used to show melanocytes above the dentate line $e^{2,5,8}$. The most up-to-date techniques use immunohystochemical reactions such as $\mathrm{S}-100$ protein (antibodies S-100), which has greater sensibility, but less specificity. Monoclonal antibodies are also used as a detection method, such as HMB-45 (monoclonal Enzo)2.

The Breslow classification is best used to classify the depth of the tumor ${ }^{1,8}$. This method is preferred to the Clark classification, regarding the lack of papillary dermis in the region ${ }^{1,8}$.

Many authors classify anorectal melanomas in stages; the most common being: stage I - in situ; stage II - regional spread (inguinal lymphadenopathy); stage III - metastasis ${ }^{1,3}$.

The metastasis areas can be arranged according to its frequency: lungs, bones, liver, brain and gastrointestinal tract ${ }^{1,3}$.

The early diagnosis is essential to improve the prognosis of such an aggressive disease. Survival rates vary from $6-12 \%$ for five years $4,7,10$. The average survival rate in literature is 18 months $^{12}$

Some factors can worsen the prognosis such as late diagnosis, ulceration, rich mucous vascularization, and aggressive nature of the tumors.

Since the most common complaints are enterorrhagia and anal bleeding, it is essential to perform a proctosigmoidoscopy and biopsy $7,8,9$. The pathologist should be reminded of the hypotheses, for there might be difficulties in differential diagnosis of anaplastic carcinoma and hemorrhoidal disease. It is essential to obtain other anatomopathological evidence. Some authors recommend using contrast exams along with colonoscopy ${ }^{3,7,12}$.

The malignant anorectal melanoma presents early metastases ${ }^{1}$. Mesenteric lymph nodes involvement is more common than inguinal ${ }^{1}$. Rectum lesions drain to the mesorectal lymph nodes and to the inferior mesenteric chain, which can occur in $33.3 \%$ of the cases. Cutaneous anal lesions drain to the superficial inguinal lymph nodes ${ }^{1}$. The dissemination through hematogenous pathway can reach the liver, lungs, bones, brain and gastrointestinal tract ${ }^{1}$. Authors diverge regarding conduct and treatment, although the recommended procedure is surgery, there are debates regarding local excision or abdominoperineal resection, and another group of authors that indicates radiotherapy and/or chemotherapy to control signs and symptoms, such as bleeding, regarding intracavitary and inguinal lymph nodes.

For some authors ${ }^{1,6}$ abdominoperineal resection can lead to a greater survival rate, especially if there is no lymph node invasion (Stage I).

The great majority of authors did not show significant difference in survival rate in patients referred to abdominoperineal resection and local excision ${ }^{1,3}$. There is some consensus on the fact that less recurrence is observed when abdominoperineal resection is performed, but with no statistical significant ${ }^{3}$.

Another aspect is that patients without compromised lymph nodes who are refered to abdominoperineal resection have a more favorable prognosis when compared to cases with compromised lymph nodes ${ }^{6}$. Some authors believe that the prognosis is strictly related to the aggressive nature and early surgery 4 .

Regarding adjuvant treatments, literature contests the success of radiotherapy (anorectal muscle non-responsiveness), as well as the lack of clarity of chemotherapy success ${ }^{4}$. Radiotherapy and hyperthermia is mentioned to control in situ disease, followed by a regression of the tumor ${ }^{12}$. Radiotherapy is not the treatment of choice, but it can be used as palliative treatment ${ }^{12}$. Immunotherapy is another method, being administered to patients that become surgically free (macroscopically) of the disease. This treatment is carried out for 4-7 months with subcutaneous injections of irradiated melanocytes with Calmette-guerin bacilli.

In conclusion, studies show the highly aggressive nature of the tumor, and the difficulty in early diagnosis and initial clinical staging. The prognosis is poor and patients face challenging metastases ${ }^{3}$. Surgical outcomes are mediocre and very few live over five years ${ }^{1}$.

\section{REFERENCES}

1. Campos FGCM, Habr-Gama A, Silva JH, Ibrahim RE, Tudor R, Pinotti $\mathrm{HW}$. Malignant melanoma of the anorectal region: report of a case and review of the literature. ABCD. Arq Bras Cirg Dig, 1989;4(4), 102-7.

2. Clemmensen OJ, Fenger C. Melanocytes in the anal canal epithelium. Histopathology. 1991 Mar;18(3):237-41.

3. Dhaliwal VS, et al. Malignant melanoma of anorectal; J. Indian M.A., 1987, 85(11), 341-3.

4. Eng J, Sabanathan S, Whittaker M. Primary anorectal malignant melanoma. Acase report. Acta ChirScand. 1989 Jun-Jul;155(6-7):357-9.

5. García Montes JM, Jiménez Sáenz M, Hernández Peña M, Herrerías Gutiérrez JM.[Anorectal melanoma]. Rev Esp Enferm Apar Dig. 1989 Aug;76(2):173-5. 
6. GoldmanS, Glimelius B, Påhlman L. Anorectal malignant melanoma in Sweden. Reportof49 patients. DisColon Rectum. 1990Oct;33(10):874-7.

7. Jorge E, Harvey HA, Simmonds MA, Lipton A, Joehl RJ. Symptomatic malignantmelanoma of the gastrointestinal tract. Operative treatment and survival. Ann Surg. 1984 Mar;199(3):328-31.

8. Kantarovsky A, Kaufman Z, Zager M, Lew S, Dinbar A. Anorecta region malignant melanoma. J Surg Oncol. 1988 Jun;38(2):77-9.

9. Knysh VI, Timofeev luM, Serebriakova ES. [Treatment of melanomas of the anorectal region]. Vopr Onkol. 1987;33(3):74-8.

10. Pyper PC, Parks TG. Melanoma of the anal canal. Br J Surg. 1984 Sep;71(9):671-2

11. Singh W, Madaan TR. Malignant melanoma of the anal canal. Am J Proctol. 1976 Feb;27(1):49-55

12. Slingluff CL Jr, Vollmer RT, Seigler HF. Anorectal melanoma: clinical characteristics and results of surgical management in twenty-four patients. Surgery. 1990 Jan;107(1):1-9.

13. Wong JH, Cagle LA, Storm FK, Morton DL. Natural history of surgically treated mucosal melanoma. Am J Surg. 1987 Jul;154(1):54-7.

\section{CENTRAL HEPATECTOMY FOR BILIARY CYSTADENOMA: PARENCHYMA-SPARING APPROACH FOR BENIGN LESIONS}

Hepatectomia central para cistoadenoma biliar: preservação de parênquima em lesões benignas

Raphael L. C. ARAUJO',2; Danielle CESCONETTO'; Vagner Birk JEISMANN ${ }^{1}$; Gilton Marques FONSECA ${ }^{1}$; Fabricio Ferreira COELHO'; Jaime Arthur Pirola KRUGER ${ }^{1}$; Paulo HERMAN,

From the ${ }^{1}$ Liver Surgery Unit, Hospital das Clínicas, University of São Paulo Medical School, São Paulo, SP; ${ }^{2}$ Department of Upper GI and HPB Surgery, Barretos Cancer Hospital, Barretos, SP, Brazil

HEADINGS - Hepatectomy. Cystadenoma DESCRITORES - Hepatectomia. Cistadenoma

\section{Correspondence:}

Raphael L. C. Araujo

E-malraphaelarajo@hcancerbarretoscomb
Financial source: none

Conflicts of interest: none

Received for publication: 03/12/2015 Accepted for publication: 02/06/2016
C entral hepatectomy $(\mathrm{CH})$ is also known as mesohepatectomy and means hepatic resection of segments 4, 5, and $8^{9}$. Hepatic lesions located in these segments may require extensive resections, such as right, left, extended right or extended left hemi-hepatectomies especially due to their relationship to major vascular and biliary structures. $\mathrm{CH}$ represents a potential risk of intraoperative bleeding, biliary injury, and risk of positive margins, but also represent the appealing concept of parenchyma sparing, furthermore in benign lesions.

Is reported a case of a symptomatic patient with a large complex cystic tumor who underwent a $\mathrm{CH}$ without tumor violation and no major postoperative complication.

\section{CASE REPORT}

A 61-year old female patient with history of choluria, acholic stools, jaundice and pain in the right upper abdominal quadrant had undergone a cholecystectomy and hepatic cyst unroofing by laparotomy in another institution, 30 months ago. Due to the cholestatic symptoms recurrence, she was refered to our center.

Abdominal MRI showed a cystic lesion in segment 4 with septa and thickened walls, and measuring $9.0 \mathrm{~cm}$. The cyst was demonstrated as isosignal on T1 and hyperintense signal on T2. The confluence of left and right bile ducts was compressed by the cyst, which caused moderate bilateral dilation. The lateral limit of the cyst compressed the left hepatic artery and the left branch of the portal vein, while its lower limit compressed the right portal branch and the right hepatic artery. Other noncomplex cystic lesions were scattered through the liver (Figure1). Laboratory tests showed increased canalicular enzymes and bilirubins and negative tumor markers. The case was reviewed at a weekly hepatobiliary multidisciplinary conference and the main hypothesis was a recurred biliary cystadenoma. In order to avoid a right trisectionectomy the decision was to perform a parenchymal preserving resection - central hepatectomy.

During surgery, was confirmed the close relationship of the cyst and the hilar plate. Intraoperative ultrasound showed compression but not invasion of the hilar plate. The liver inflow was controlled with intrahepatic pedicle ligation of right anterior sector and segment 4 . The cyst was separated from the hilar plate using ultrasonic dissector and bipolar eletrocoagulation, as demonstrated in Figures 2 and 3. Parenchyma transection was carried out with intermittent pedicle clamping (Pringle's maneuver). No blood transfusion was necessary. The postoperative course was only marked by a low volume biliary fistula conservatively managed with cavity drain placed during surgery (grade I - Dindo \& Clavien classification $)^{2}$. She was discharged on $8^{\text {th }}$ postoperative day. Pathological examination revealed a biliary cystadenoma presenting low-grade neoplasia with free margins. After 18 months of follow-up, the patient is doing well without either symptomatic or radiological recurrence (Figure 4).

\section{DISCUSSION}

$\mathrm{CH}$ is also known as mesohepatectomy, central hepatic resection, middle hepatectomy, middle hepatic lobectomy, and central bisectionectomy ${ }^{9}$. The putative risks of it compared to traditional major liver resections include a longer procedure time, greater intraoperative blood loss, higher risk of biliary and vascular complications, all mainly attributed to the proximity to hilar structures and the presence of two significant resection planes instead of a single one. Despite those concerns, this case highlighted that $\mathrm{CH}$ is safe and can be accomplished without significant morbidity. No significant differences for postoperative morbidity and mortality between $\mathrm{CH}$ and extend hepatectomy $(\mathrm{EH})$ were demonstrated by Lee's systematic review ${ }^{4}$. Additionally, a recent case-matched study from the same author showed no differences in 90-day mortality, biliary leaks and postoperative liver failure ${ }^{4}$. Moreover, this study also showed longer length of stay, higher postoperative bilirubin and longer prothrombine time for patients who underwent extend hepatectomy.

Liver parenchyma sparing aims to decrease the risk of postoperative liver failure, shorten recovering time, and allow re-hepatectomies in patients with high risk of recurrence. In patients with multifocal benign (adenomatosis) or malignant 